\title{
Guest Editorial Channel Coding in Wireless Systems
}

\begin{abstract}
I $\mathrm{t}$ is our pleasure to introduce this Special Issue on 1 "Channel Coding in Wireless Systems" of the Journal of Communication Software and Systems (JCOMSS).

This is one of the initiatives conceived to promote JCOMSS, a relatively new journal born from an idea of the Croatian Communication and Information Society (CCIS) and the University of Split, and endorsed by the IEEE Technical Committee on Communications Software of the IEEE Communications Society.

Really, the Croatian group is very active in supporting IEEE activities and proposing original ideas for exchanging experiences and results among researchers coming from universities, industries and research centers. Just to mention an example, it is the main organizer of SoftCOM, the International Conference on Software, Telecommunications and Computer Networks, that is probably the only ICT workshop in the world to be held aboard a ship, cruising along the Croatian and the Italian coasts. This year SoftCOM will celebrate its $14^{\text {th }}$ edition.
\end{abstract}

Then, charmed from so much dynamism, when Prof. Nikola Rozic asked for us to organize a special issue on the theoretical and practical aspects of channel coding for wireless applications, we accepted the invitation with great enthusiasm.

Wireless systems are a privileged field to discuss the advantages of channel coding in telecommunications. The wireless channel is a particularly severe test-bed: it is affected by nonlinearities, multipaths, Doppler shifts, fading, shadowing, interference from other users, and many other impairments depicting an involved scenario, difficult to treat but also exciting for proposing new and attractive solutions. The invention of turbo codes in 1993 has been followed by the application of iterative techniques to many other blocks of communication systems, and the rapid implementation of these concepts in practical applications. In the last ten years new schemes have been designed (and old ones reinterpreted) able to approach Shannon capacity limits with reasonable complexity. This way, new applications and services become possible, quite unthinkable in the past.

Our goal was to realize a special issue representing a useful starting point for young researchers and $\mathrm{PhD}$ students interested in modern channel coding. For this reason we invited some universally recognized experts to write a semi-tutorial paper describing the state-of-the-art, the recent evolution, and the main open issues for three key coding subjects: Claude Berrou (turbo codes), Bill Ryan and Shu Lin (LDPC), and Giuseppe Caire (space-time codes). We are really indebted with them because, as the reader will find, they have written three excellent papers, containing both a perfect compendium on their subject and new important results.

So, it is an honor to start the volume with a paper by Claude
Berrou, the inventor of turbo-codes and the main responsible for the coding revolution. In his paper "Turbo codes and turbo algorithms", co-authored by Charlotte Lanlglais and Yi Yu, Prof Berrou provides a clear and explicit discussion of the turbo principle and demonstrates the importance to have a bi-directional message passing for approaching almost ideal performance. He also introduces for the first time the novel concept of genie-initialized iterative processing (GIIP) as a tool to assess the error performance of iterative processing. Finally, he applies the new method to two examples of relevant importance for wireless systems, that is MultipleInput/Multiple-Output (MIMO) and Linear Precoding.

The second invited paper, entitled "Design of LDPC codes: a survey and new results", has been written by Bill Ryan, Shu Lin, Gianluigi Liva, Shumei Song, Lan Lan and Yifei Zhang. It provides an excellent survey of the design techniques used for these codes, with special emphasis on those yielding cyclic and quasi-cyclic codes, because of the benefits they offer in terms of reduced encoding complexity and decoding efficiency. Several algebraic constructions are considered and rather new concepts, like that of protographs or Reed-Solomon based LDPC codes, are highlighted and their features discussed. Moreover, the paper includes a number of performance results and codes structures not yet presented elsewhere.

The third paper "Space-time coding: an overview", written by Giuseppe Caire, Petros Elias and K. Raj Kumar, deals with space-time codes and their natural application in wireless systems, where they improve performance of diversity techniques based on multiple antennas. The paper gives a comprehensive overview of the fundamental aspects in spacetime coding, starting from classic information theoretic results on multiple-input multiple-output fading channels and then discussing the fundamental issue of optimal codes design. In particular, they review a number of constructions that permit to achieve the so-called optimal diversity-multiplexing tradeoff, justifying the approach by means of an original theory through which the authors have been able to recently extend the design procedures of these codes.

The special issue is completed by 4 regular papers, selected after international review process. They discuss different aspects of channel coding in wireless system, all relevant from the theoretical or practical point of view.

In "Turbo codes performance optimization over block fading channels", Fulvio Babich, Guido Montorsi and Francesca Vatta find the best adaptation of a turbo code to a block fading channel. Code adaptation is achieved through the insertion of an interleaver, following the turbo encoder, that spreads the symbols on different subchannels, thus providing an amount of diversity. By rigorous arguments from the information theory, they are able to find the nearly best achievable performance of 
a turbo code over a block fading channel, that is a remarkable result for wireless applications.

In "Some extended results on the design of punctured serially concatenated convolutional codes", Massimiliano Laddomada and Bartolo Scanavino show the results of an exhaustive search for optimum constituent encoders and puncturing patterns to apply in this class of codes. Either the waterfall region or the error floor region, are taken into account in the analysis, that therefore provides very general design tools.

Roberto H. Morelos-Zaragoza has written "On the error performance of coding and equalization in low-complexity ultra-wideband communication systems", where he analyzes pulse-based ultra-wideband (UWB) communication systems for applications in short-range indoor environments. On these systems, various channel coding schemes are applied and compared, also including decision feedback equalization. The paper highlights, among the others, the class of Turbo Product Codes (TPCs), as they offer the best tradeoff between error performance and decoding complexity for the considered application and the bit error rates of interest.

Finally, Maurizio Martina, Andrea Molino, Fabrizio Vacca, Guido Masera and Guido Montorsi, in the paper entitled "High throughput implementation of an adaptive serial concatenation turbo decoder", face the fundamental problem of practical implementation of a turbo encoder/decoder incorporating the relevant, and difficult to meet, issues of high data rates and flexibility. The authors give exhaustive implementation details, proving feasibility in a commercial single Field Programmable Gate Array (FPGA) device, with very high throughput and limited percentage device occupation, taking benefits, for this purpose, by highly regular and parallel structures. At the end of this introduction, thanks to the value of the authors and the importance of the subjects discussed, we hope to have been able to prepare a good special issue, useful for channel coding researchers. If this will be, the major merits, of course, shall be attributed to the authors themselves, and for this reason we wish to thank all of them once again.

31. August 2006

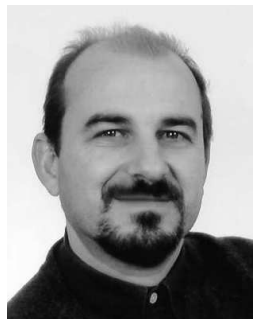

Marco Chiani was born in Rimini, Italy, in April 1964. He received the Dr. Ing. degree (magna cum laude)in Electronic Engineering and the Ph.D. degree in Electronic and Computer Science from the University of Bologna in 1989 and 1993, respectively. Dr. Chiani is a Full Professor at the II Engineering Faculty, University of Bologna, Italy, where he is the Chair in Telecommunication. During the summer of 2001 he was a Visiting Scientist at AT\&T Research Laboratories in Middletown, NJ. He is a frequent visitor at the Massachusetts Institute of Technology (MIT), where he presently holds a Research Affiliate appointment.

Dr. Chiani's research interests include wireless communication systems, MIMO systems, wireless multimedia, low density parity check codes (LDPCC) and UWB. Under the European research program PROMETHEUS he has worked on short-range millimeter wave communication systems for Advanced Road Transport Telematics. He is also leading the research unit of CNIT/University of Bologna on Joint Source and Channel Coding for wireless video (IST FP6, project Phoenix) and is a consultant to the European Space Agency (ESA-ESOC) for the design and evaluation of error correcting codes based on LDPCC for space CCSDS applications. Prof. Chiani has been a consultant for several other telecommunications industries and operators, and engages in active collaboration with many research centers and universities, such as MIT, NASA/JPL, VTT, Thales, Philips, Telecom New Zealand, the University of Canterbury, and the University of Arizona.

Dr. Chiani is actively involved in various communications research activities worldwide. He is the past chair (2002-2004) of the Radio Communications Committee of the IEEE Communication Society and the current Editor of Wireless Communication for the IEEE Transactions on Communications. Dr. Chiani has chaired, organized sessions and served on the Technical Program Committees at several IEEE International Conferences. He was Co-Chair of the Wireless Communications Symposium at ICC 2004. In January 2006 he received the ICNEWS award "For Fundamental Contributions to the Theory and Practice of Wireless Communications".

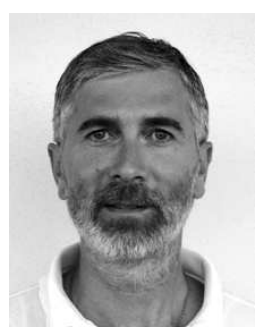

Franco Chiaraluce was born in Ancona, Italy, in 1960. He received the "Laurea in Ingegneria Elettronica" (summa cum laude) from the Universitá di Ancona in 1985. Since 1987 he joined the Dipartimento di Elettronica ed Automatica of the same university. At present, he is an Associate Professor at the Universitá Politecnica delle Marche. His main research interests involve various aspects of communication systems theory and design, with special emphasis on coding, cryptography and multiple access techniques. In the specific field of error correcting codes he co-operates with national and international telecommunications companies, and has been also involved in activities with the European Space Agency (ESA), under the framework of joint researches or contracts. He is co-author of more than 170 papers and two books. He is member of IEEE and IEICE.

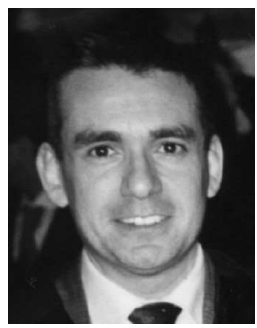

Roberto Garello was born in Torino, Italy, on December 18, 1965. He received the "Laurea "; degree (summa cum laude) in 1989 and the "Dottorato di Ricerca" degree in electronic engineering in 1994, both from Politecnico di Torino. During 1993, he spent six months visiting the Laboratory for Information and Decision Systems, the Massachusetts Institute of Technology (MIT), Cambridge, and the Signal and Information Processing Laboratory, ETH, Zurich. From October 1994 to September 1997, he was a Design Engineer at the Radio Link Laboratory of Marconi Communications, Genova, working on high-rate all-digital modems and error-control scheme design. From November 1998 to October 2001 he was an Associate Professor at the Dipartimento di Elettronica ed Automatica of University agrave; di Ancona, Italy. Since November 2001 he is an Associate Professor at the Dipartimento di Elettronica of Politecnico di Torino, where he currently serves as coordinator of the Telecommunication Engineering degree. Senior member of IEEE, his main research interests are coding theory and genomic signal processing.

\section{ROBERTO GARELLO} Politecnico di Torino Italy

\author{
Universita Politecnica delle Marche \\ Ancona, Italy \\ Universita di Bologna \\ Italy
}

